\title{
Prevalencia de Nosema spp. (Microsporidia: Nosematidae) en abejas africanizadas en Atenas, Costa Rica: número de esporas y nivel de infección durante la época lluviosa
}

\section{Prevalence of Nosema spp. (Microsporidia: Nosematidae) in Africanized Honeybees in Atenas, Costa Rica: Number of Spores and Infection Level During the Rainy Season}

\section{Prevalência de Nosema spp. (Microsporidia: Nosematidae) em abelhas africanizadas em Atenas, Costa Rica: número de esporos e nível de infecção durante a estação chuvosa}

Eduardo Moreno $^{1}$; Rafael A. Calderón ${ }^{1 凶}$; Marianyela Ramírez ${ }^{1}$; Johan Van Veen ${ }^{2}$

1 Programa Integrado de Patología Apícola, Centro de Investigaciones Apícolas Tropicales, Universidad Nacional, Heredia,

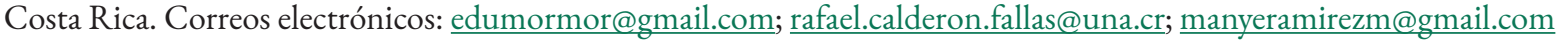

2 Programa Regional de Apicultura y Meliponicultura, Centro de Investigaciones Apícolas Tropicales, Universidad Nacional, Heredia, Costa Rica. Correo electrónico: johan.vanveen.marinissen@una.cr

Recibido: 16 de octubre de 2020 Corregido: 12 de abril de 2021 Aceptado: 15 de abril de 2021

\begin{abstract}
Resumen
Se estudió la prevalencia del microsporidio Nosema spp. en un apiario de 10 colmenas de abejas africanizadas en AtenasAlajuela, Costa Rica, durante julio a noviembre 2017. Además, se comparó el número de esporas y el nivel de infección en muestras grupales e individuales de abejas adultas provenientes de una misma colmena. Se muestreó el apiario con un intervalo de 30 días. De cada colmena se colectaron aproximadamente 100 abejas adultas de la piquera, de las cuales 30 se examinaron de manera grupal y 30 individualmente en el Laboratorio de Patología Apícola-CINAT. Para determinar las esporas de Nosema spp. las abejas se analizaron mediante el método de Cantwell. Para el análisis grupal, se tomaron 30 abejas y se procedió a cortar los abdómenes, los cuales se maceraron en un mortero, agregando $30 \mathrm{ml}$ de agua destilada. En el examen individual, se utilizó la misma técnica y se analizó cada abeja por separado. La muestra se revisó en el microscopio a un aumento de 40x, se procedió a identificar y determinar el número de esporas mediante el hemocitómetro y a establecer el nivel de infección de cada colmena. Se determinó la presencia de Nosema spp. en todas las colmenas del apiario, pero con diferentes niveles de infección, variando desde leve hasta fuerte, tanto en el análisis grupal como en el individual. En julio se cuantificó el mayor número de esporas, con un promedio de 20,360,000 $\pm 1,586,957$ por abeja en el examen grupal y 12,749,733 \pm 867,232 por abeja en el individual. En los meses siguientes se observó un descenso considerable en el número de esporas, hasta contabilizar la menor cantidad en noviembre, con un promedio de 4,375,000 $\pm 874,132$ y 2,087,708 $\pm 398,895$ para los análisis grupal e individual, respectivamente. Al comparar el conteo de esporas y el nivel de infección de Nosema spp. en muestras grupales e individuales, se obtuvieron resultados similares. Además, al correlacionar la cantidad de esporas se estableció una alta proximidad en los valores, mostrando que mediante ambos métodos se obtiene un resultado semejante en el nivel de infección de la misma colmena.
\end{abstract}

Palabras clave: Nosema spp., nosemosis, nivel de infección, época lluviosa, abejas africanizadas

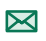

Autor de correspondencia:rafael.calderon.fallas@una.cr 


\begin{abstract}
The prevalence of the microsporidium Nosema spp. was studied during the rainy months from July to November 2017 in 10 Africanized honeybees colonies in Atenas-Alajuela, Costa Rica. In addition, the number of spores and the infection level were compared in group and individual samples of adult bees from the same colony. The apiary was sampled once a month. Approximately 100 forager bees per colony were collected, of which 30 were examined for Nosema spp. in group analysis and 30 individually at the Bee Pathology Lab-CINAT. According to the Cantwell method, the abdomens of 30 adult bees were cut and macerated together with $30 \mathrm{ml}$ of distilled water for the group test. For the individual analysis, the same method was used, but each bee was examined separately. Nosema spores were identified under the cover slip using a light microscopy at 40x magnification and were counted with a hemocytometer. The infection level was determined for every colony. The microsporidium Nosema spp. were found in all apiary colonies at different levels, ranging from low to severe infection in both group and individual exams. In July, the highest number of Nosema spores were quantified, with an average of 20,360,000 $\pm 1,586,957$ per bee in the group analysis, and 12,749,733 $\pm 867,232$ per bee in the individual analysis. In the following months, a considerable decrease in the number of spores was observed, and the lowest amount was recorded in November, with an average of $4,375,000 \pm 874,132$ and 2,087,708 $\pm 398,895$ spores per bee in the group and individual exam, respectively. When comparing the spore count and the infection level of Nosema spp. in group and individual samples, similar results were obtained. Furthermore, by correlating the number of spores, high proximity in the values was found, showing that, by both methods, a similar result is obtained concerning the infection level of the same colony.
\end{abstract}

Keywords: Nosema spp., nosemosis, infection level, rainy season, africanized honeybees

\title{
Resumo
}

A prevalência do microsporídio Nosema spp. foi estudado durante os meses de julho a novembro de 2017, em 10 colmeia de abelhas africanizadas em Atenas-Alajuela, Costa Rica. Além disso, o número de esporos e o nível de infecção foram comparados em grupos e amostras individuais de abelhas adultas da mesma colmeia. Realizaramse amostragens do apiário com um intervalo de 30 dias. Em cada colmeia, foram coletadas aproximadamente 100 abelhas adultas, das quais 30 foram examinadas em análise de grupo e 30 individualmente, no Laboratório de Patologia Apícola-CINAT. Para determinar os esporos de Nosema spp. as abelhas foram analisadas pelo método de Cantwell. Para a análise do grupo, foram retiradas 30 abelhas cujos abdomens foram cortados e macerados, acrescentando-se $30 \mathrm{ml}$ de água destilada. Para a análise individual, o mesmo método foi usado, mas cada abelha foi examinada separadamente. A amostra foi analisada ao microscópio com aumento de 40x. O número de esporos foi identificado e determinado com o hemocitômetro e o nível de infecção em cada colmeia foi estabelecido. $\mathrm{O}$ microsporídio Nosema spp. foi encontrado em todas as colmeias do apiário, em diferentes níveis de infeccção, variando de leve a forte, tanto nas análises grupais como nas individuais. Em julho, foi quantificado o maior número de esporos, com uma média de 20.360.000 \pm 1.586 .957 por abelha na análise de grupo e $12.749 .733 \pm 867.232$ por abelha, no individual. Nos meses seguintes, observou-se uma diminuição considerável no número de esporos, sendo a menor quantidade registrada em novembro, com uma média de $4.375 .000 \pm 874.132$ e $2.087 .708 \pm 398.895$ esporos por abelha para análises grupais e individuais, respectivamente. Ao comparar a contagem de esporos e o nível de infecção de Nosema spp. em grupos e amostras individuais, resultados semelhantes foram obtidos. Além disso, ao correlacionar o número de esporos, encontrou-se uma alta proximidade nos valores, mostrando que, por ambos métodos, um resultado semelhante é obtido em relação ao nível de infecção da mesma colmeia.

Palavras-chave: Nosema spp., Nosemose, nível de infecção, estação chuvosa, abelhas africanizadas

\section{Introducción}

Se han descrito más de 35 enfermedades asociadas a las abejas melíferas, Apis mellifera, la mayoría de ellas ocasionan daños considerables en la apicultura (Foley et al., 2014; Ritter, 2001). Una de las enfermedades 
de mayor importancia económica a nivel mundial es la nosemosis (Fries, 2010; Hamiduzzaman et al., 2015; Muñoz et al., 2014;). Esta enfermedad es causada por dos especies de microsporidios, Nosema apis y Nosema ceranae, ambas forman esporas que infectan las células epiteliales del intestino de las abejas adultas (Botías et al., 2012; Bravo et al., 2014; Jara et al., 2015; Muñoz et al., 2014; Traver \& Fell, 2011). Recientemente, se reportó en Uganda una nueva especie, Nosema neumanni, de la cual se tiene poca información (Chemurot et al., 2017). En Costa Rica, la nosemosis causada por $N$. apis, fue descrita por primera vez en 1985, mientras que en el 2006 se detectó $N$. ceranae al analizar muestras de abejas adultas provenientes de diferentes zonas apícolas del país (Calderón et al., 2008; Calderón \& Ramírez, 2010).

Según Eiri et al. (2015) la infección por Nosema spp. provoca degeneración del tejido digestivo, lo que ocasiona desnutrición. Además, afecta el comportamiento de vuelo y genera pérdida de la orientación en abejas pecoreadoras, lo cual reduce de manera paulatina la cantidad de obreras y provoca el despoblamiento de la colonia (Higes et al., 2007). En casos severos, se observan abejas con el abdomen abultado, marcas fecales color marrón en los panales y en la parte frontal de la colmena, abejas que no pueden volar y abejas muertas en el suelo frente a la piquera (Calderón \& Sánchez, 2011). Además, las abejas nodrizas infectadas disminuyen la producción de jalea real y la reina reduce la postura de huevos (Chihu et al., 2013). Lo anterior, origina pérdidas significativas en la producción de miel y serias deficiencias en los servicios de polinización que brindan las abejas (Copley et al., 2012). Esta enfermedad puede permanecer latente durante todo el año y manifestarse después de periodos de hacinamiento de las abejas dentro de la colmena, debido a lluvias persistentes, fríos intensos o fuertes vientos, ya que estas condiciones favorecen el contacto y contagio entre las abejas (Calderón \& Ramírez, 2010).

El procedimiento tradicional para el diagnóstico de la nosemosis en el laboratorio consiste en el examen de una muestra grupal de 30 a 60 abejas adultas por colmena, lo cual permite obtener un estimado del número de esporas por abeja (Botías et al., 2012). Sin embargo, se ha reportado que, al aplicar esta metodología, en ciertos casos podría obtenerse un diagnóstico poco confiable, ya que existe variación en la intensidad de la infección entre individuos de una misma colmena, por lo que se podría sobreestimar o subestimar el nivel de infección (Mulholland et al., 2012). Algunos autores mencionan que el análisis individual de abejas es un indicador más certero de la nosemosis en la colonia. No obstante, los estudios sobre Nosema spp. en abejas individuales, son escasos (Higes et al., 2008; Mulholland et al., 2012).

Debido a la poca investigación relacionada con el microsporidio Nosema spp. en abejas africanizadas bajo condiciones tropicales, específicamente el análisis de abejas individuales, se realizó el presente estudio con el objetivo de comparar la cantidad de esporas y el nivel de infección de una muestra grupal con el análisis individual de abejas adultas provenientes de la misma colonia. Lo anterior, permite tener un resultado más confiable de la intensidad de la infección de este microsporidio en las colmenas y de esta manera definir estrategias de control en el apiario.

\section{Materiales y métodos}

El estudio se realizó en un apiario constituido por 10 colmenas de abejas africanizadas (A. mellifera) (Spivak, 1991), ubicado en Río Grande, distrito de Concepción- (0957’45” N, 84²1’50” O, 535 msnm), cantón de Atenas-Alajuela, Costa Rica. La región de Atenas se clasifica como bosque húmedo premontano, con una temperatura promedio anual de $25^{\circ} \mathrm{C}$ y una precipitación entre 1011 y $2022 \mathrm{~mm}$ (IMN 2017). La investigación se llevó a cabo de julio a noviembre de 2017 (época lluviosa), meses en los cuales se ha reportado 
una mayor incidencia de nosemosis (Calderón \& Ramírez, 2010; Calderón \& Pichardo, 2011). La mayoría de las colmenas estaban conformadas por ocho panales (con cría y reservas de miel/polen) y no se trataron contra ninguna enfermedad en los meses previos al estudio (enero-junio 2017). Además, se confirmó la presencia de esporas de Nosema spp. mediante análisis de laboratorio de las abejas adultas (método de Cantwell), las cuales adquirieron el microsporidio naturalmente.

\section{Colecta de muestras}

Se muestreó el apiario con un intervalo de 30 días. De cada colmena se colectaron aproximadamente 100 abejas de la piquera (entrada de la colmena), con el fin de evaluar abejas pecoreadoras (con más de 21 días de edad) y de esta forma evitar "falsos negativos" (OIE, 2013; Pichardo et al., 2012). Existen reportes que indican que las abejas pecoreadoras tienen mayores niveles de infección de Nosema spp. en comparación con las abejas nodrizas presentes en el nido de cría (Higes et al., 2008; Higes et al., 2010; Medina et al., 2014). La piquera de la colmena se cerró temporalmente con papel periódico, durante unos 20 - 30 minutos en horas de la mañana (9:00 am - 11:00 am) (Higes et al., 2008; Botías et al., 2012). Cuando se observó un número considerable de abejas agrupadas en la entrada, se colectaron con una aspiradora para insectos y se colocaron en un frasco con alcohol etílico al 70.0\%.

\section{Análisis}

Las muestras de abejas se analizaron en el Laboratorio de Patología Apícola del Centro de Investigaciones Apícolas Tropicales (CINAT-UNA). Para determinar la presencia y cantidad de esporas de Nosema spp., las abejas se examinaron mediante el método de Cantwell (Molina et al., 1990). De cada muestra se tomaron 30 abejas, las cuales se colocaron sobre un papel absorbente y se procedió a retirar los abdómenes, los cuales se maceraron en un mortero y se añadió $1.0 \mathrm{ml}$ de agua destilada por cada uno de ellos (total $30.0 \mathrm{ml}$ ). El macerado se homogenizó agitándolo constantemente durante un minuto y se colocó una gota de la suspensión en un portaobjetos. Para identificar el microsporidio, la muestra se revisó en el microscopio a un aumento de 40x. Las esporas se identificaron por ser ovaladas, brillantes y refringentes.

Al observar las esporas de Nosema spp. con la técnica de Cantwell, se procedió a cuantificarlas y determinar el nivel de infección de cada colmena mediante el hemocitómetro (Calderón \& Sánchez, 2011). Se realizó el análisis grupal de 30 abejas y el examen individual de 30 abejas, provenientes de la misma muestra (colmena).

\section{Nivel de infección de Nosema spp. en una muestra grupal}

Para cuantificar las esporas en una muestra grupal de abejas, el líquido sobrenadante se agitó de manera mecánica para homogenizarlo y con una micropipeta se tomó una alícuota de $0.1 \mathrm{~mm}^{3}$, la cual se colocó en el hemocitómetro y se dejó reposar durante tres minutos, para la sedimentación de las esporas. La muestra se observó en el microscopio a 40x y se contaron las esporas de cinco bloques del hemocitómetro, los cuatro de las esquinas y el bloque del centro (Molina et al., 1990; OIRSA, 1990).

Para establecer la intensidad promedio (abundancia de esporas) de la infección se utilizó la siguiente fórmula:

Número de esporas por abeja $=($ total de esporas contadas $/ 80) \times 4,000,000$ 


\section{Nivel de infección de Nosema spp. en abejas individuales}

Para analizar el nivel de infección de Nosema spp. en 30 abejas por colmena ( $\mathrm{n}=10$ colmenas), se utilizó la técnica del hemocitómetro. Se examinó cada una de las abejas de manera individual ( $\mathrm{n}=300$ abejas por mes), macerando el abdomen y agregando $1.0 \mathrm{ml}$ de agua destilada. Posteriormente, se colocó $10 \mu \mathrm{l}$ del líquido sobrenadante en el hemocitómetro, se observó en el microscopio a 40x y se contaron las esporas. Para determinar el nivel de infección, se utilizó la fórmula indicada anteriormente. El procedimiento se repitió hasta obtener el nivel de infección de cada una de las 30 abejas analizadas individualmente por colmena.

\section{Nivel de infección}

Para categorizar la intensidad del nivel de infección de la nosemosis, el número de esporas de cada muestra (grupal e individual), se comparó con la tabla de Jaycox (Cuadro 1) (OIRSA, 1990).

Cuadro 1. Intensidad de la infección de nosemosis en abejas melíferas (Jaycox).

\begin{tabular}{ccc}
\hline Nivel de infección & Intensidad & Cantidad de esporas por abeja \\
\hline Nulo & 0 & Menos de 10,000 \\
Muy leve & 1 & $10,000-1,000,000$ \\
Leve & 2 & $1,000,001-5,000,000$ \\
Moderado & 3 & $5,000,001-10,000,000$ \\
Semifuerte & 4 & $10,000,001-20,000,000$ \\
Fuerte & 5 & Superior a 20,000,000 \\
\hline
\end{tabular}

\section{Análisis estadístico}

Los datos de la cantidad de esporas obtenidas en el examen grupal de abejas se digitaron en una tabla de Microsoft Excel $^{\circledR}$, la cual se ingresó al software Minitab ${ }^{\circledR}$, siendo clasificados por el número de colmena y el mes de evaluación. De manera similar, se digitó el número de esporas de abejas individuales, incluyendo como variables el número de abeja, el número de colmena y el mes. En este caso, se obtuvo el promedio de esporas en abejas individuales por colmena. Para la cantidad de esporas se calcularon el promedio y medidas de variación (desviación estándar y error estándar) por mes y para cada método. El promedio de esporas de muestras grupales se correlacionó con el promedio presente en abejas individuales por colmena, mediante el gráfico de dispersión y se calculó el coeficiente de correlación lineal de Pearson por mes. Los datos de precipitación, humedad y temperatura del cantón de Atenas durante el estudio se obtuvieron en el Instituto Meteorológico Nacional (IMN, 2017).

\section{Resultados}

En la presente investigación se evaluó la prevalencia y el nivel de infección del microsporidio Nosema spp. en colmenas de abejas africanizadas en Atenas-Alajuela. Las condiciones climáticas promedio en la zona durante julio a noviembre correspondieron a $24^{\circ} \mathrm{C}$ de temperatura, $89 \%$ de humedad relativa y $364 \mathrm{~mm}$ de precipitación. 


\section{Nosemosis en muestras grupales de abejas}

Se determinó la presencia del microsporidio Nosema spp. en todas las colmenas analizadas ( $\mathrm{n}=10)$, durante la época lluviosa, con diferentes niveles de infección, desde leves hasta fuertes. En julio se estableció la mayor cantidad de esporas en el apiario, con un promedio de 20,360,000 $\pm 1,586,957$ por abeja. En el siguiente mes, la infección fue significativamente inferior ( $\mathrm{p}<0.0001)$. En setiembre, se cuantificaron $6,230,000 \pm 1,396,945$, mientras que en octubre ocurrió un leve incremento. Finalmente, en el mes de noviembre, se obtuvo la menor cantidad de esporas, con un promedio de 4,375,000 \pm 874,132 (Figura 1). No se encontraron diferencias significativas entre los meses de agosto a noviembre (ANOVA= Prueba de Tukey - significancia 0.05).

\section{Esporas de Nosema spp. en abejas individuales}

Se observó la presencia de Nosema spp. en abejas individuales, con niveles de infección desde leves hasta semifuertes. En el mes de julio se contabilizó la mayor cantidad de esporas, con un promedio de 12,749,733 $\pm 867,232$ por abeja, siendo mayor que los siguientes meses, en los cuales la infección por nosemosis fue menor a 8,000,000. En agosto se cuantificó la segunda cifra más alta de esporas, de 7,367,267 $\pm 716,697$ por abeja. Durante setiembre y octubre el promedio continuó disminuyendo, hasta noviembre donde se registró la menor cantidad, 2,087,708 $\pm 398,895$ de esporas por abeja (Figura 1).

\section{Comparación del número de esporas entre muestras grupales e individuales}

Al comparar la cantidad promedio de esporas de Nosema spp. obtenidas durante los meses de muestreo mediante los métodos grupal e individual, se observó una similitud en la tendencia del resultado (aun cuando la cantidad de esporas fue mayor en el análisis grupal). Durante julio se determinó con ambos métodos la mayor cantidad de esporas, con una tendencia a disminuir en los siguientes meses (Figura 1). La mayor variación en el número de esporas entre los métodos se observó en el mes de julio con una diferencia de 7,610,267 por abeja, mientras que en setiembre se estableció la menor diferencia, siendo de 1,380,024. En octubre, el análisis grupal presentó un leve incremento en el conteo de esporas, mientras que en el individual continuó disminuyendo. En noviembre, se registró la cifra más baja de esporas en ambos exámenes, con menos de 5,000,000 por abeja (Figura 1).

\section{Comparación del valor medio de esporas}

Al analizar las medias del conteo de esporas de Nosema spp. en muestras grupales e individuales, se encontraron diferencias significativas entre ellas durante el mes de julio (significancia de 0.05). En este mes no se traslaparon los ejes de ambos intervalos, por lo que existió diferencias entre las medias, las cuales correspondieron a 20,360,000 \pm 1,586,957 de esporas en el examen grupal y 12,749,733 \pm 867,232 en el individual (Figura 2). En los meses restantes (agosto-noviembre) los intervalos de los ejes coincidieron, indicando que no existen diferencias significativas respecto al número de esporas. En este caso los promedios fueron 7,248,649 \pm 965,186 de esporas en el grupal y 4,853,288 \pm 293,620 en el individual. 


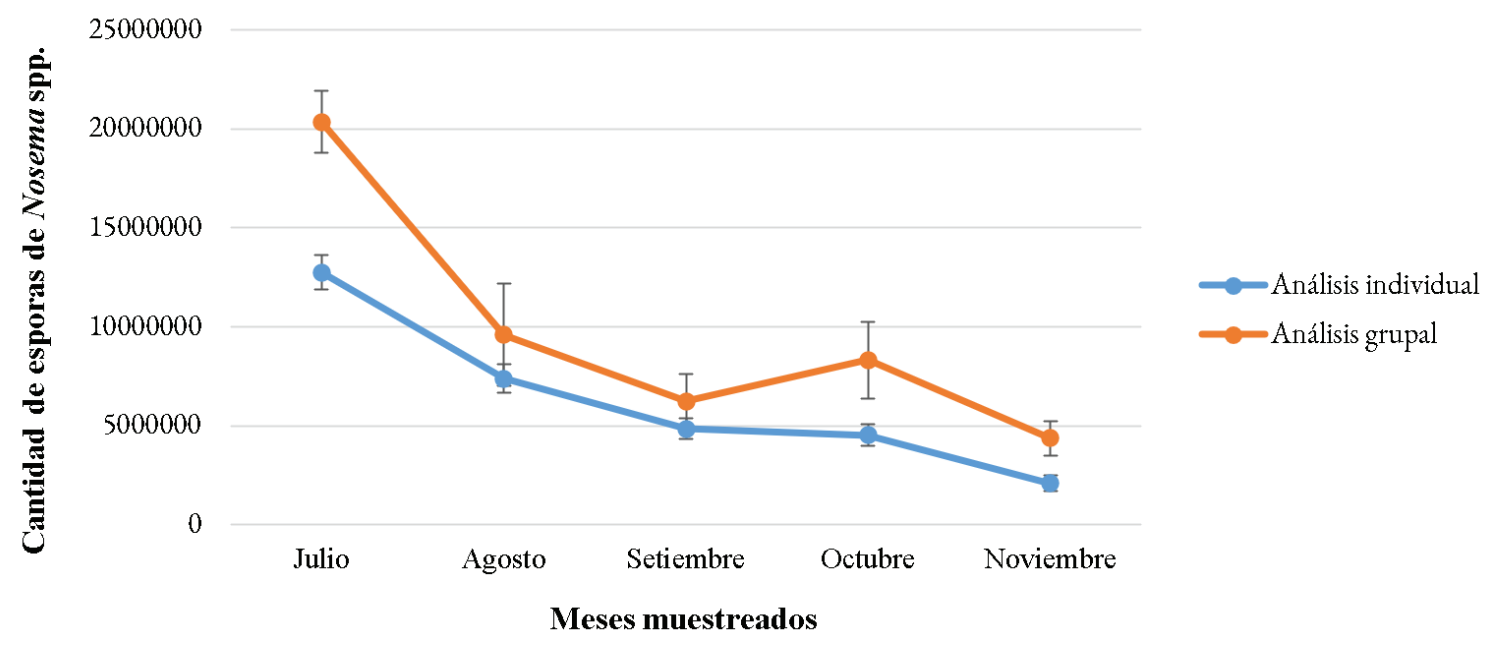

Figura 1. Número promedio de esporas de Nosema spp. al analizar muestras grupales e individuales de abejas obreras adultas durante los meses de la época lluviosa (julio-noviembre) ( $\bar{x} \pm \mathrm{SE}$ ). Elaboración propia.

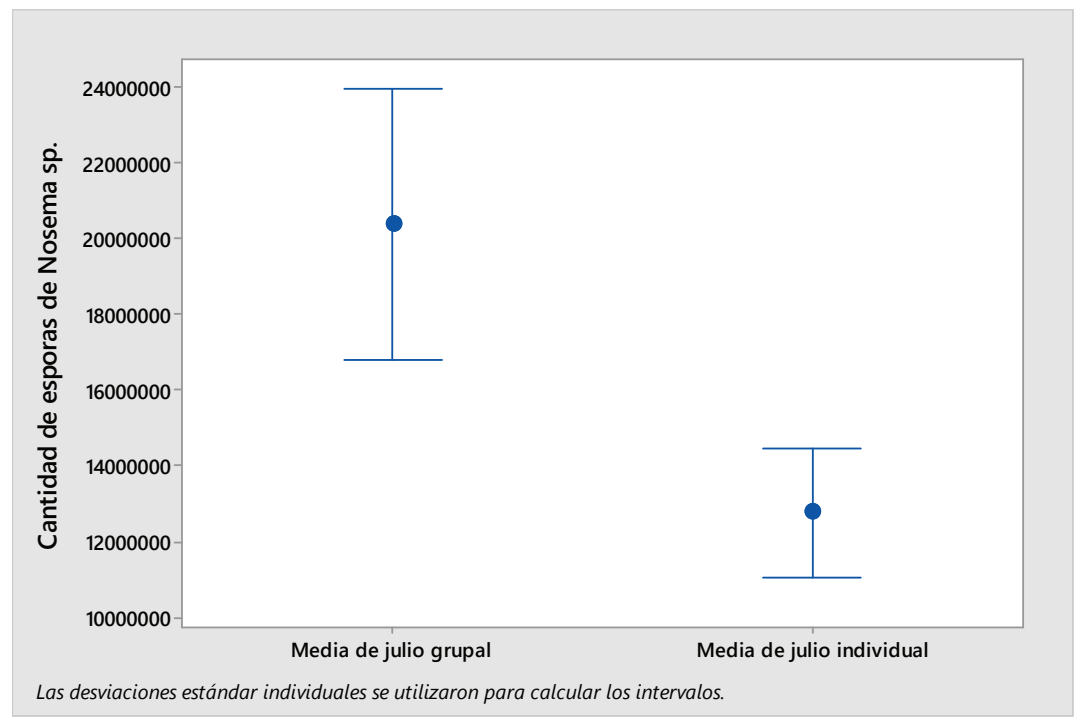

Figura 2. Comparación del valor medio del número de esporas de Nosema spp. determinadas en muestras grupales e individuales de abejas adultas durante el mes de julio (se encontraron diferencias significativas, significancia de 0.05 ). Elaboración propia.

\section{Conteo de esporas y nivel de infección de Nosema spp. por colmena por mes}

Al examinar la cantidad de esporas por colmena por mes, se observó que en julio hubo una cantidad superior a los 5,000,000 en todas las colmenas, tanto en el análisis grupal como en el individual. En el método grupal las 
colmenas presentaron niveles de infección semifuerte y fuerte, mientras que en el individual el 70.0\% mostró esos niveles. Específicamente, la colmena 36 presentó la mayor similitud en el número de esporas, el conteo grupal fue de 26,500,000 y el individual correspondió a 25,212,000 \pm 3,869,147 por abeja, clasificándose ambos como fuerte. Para cada colmena se indica el número de abejas individuales que mostraron el mismo nivel de infección que la muestra grupal. Por ejemplo, en la 36 la mitad de las abejas coincidieron con el nivel de infección del examen grupal (Figura 3).

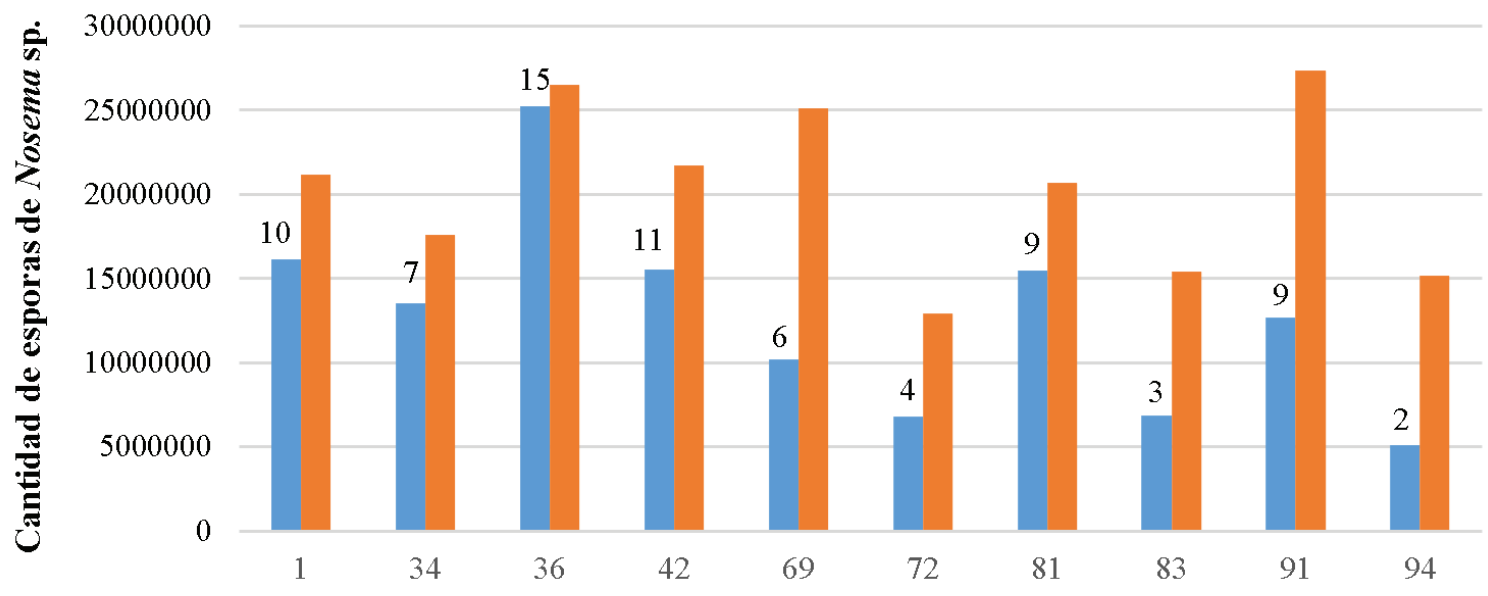

Colmenas muestreadas en julio

घnálisis individual $\square$ Análisis grupal

Figura 3. Conteo de esporas y nivel de infección de Nosema spp. mediante el examen individual (promedio de 30 conteos individuales) y grupal (valor promedio de muestra compuesta de 30 individuos) de abejas obreras durante julio para cada colmena analizada. Se indica la cantidad de abejas individuales (número sobre la barra) que presentaron el mismo nivel de infección (según Jaycox) que la muestra grupal. Elaboración propia.

Por otro lado, en agosto los niveles de infección determinados mediante ambos métodos se clasificaron desde leves hasta fuertes. En ocho de las colmenas, la intensidad de la infección fue similar. A diferencia del mes anterior, en el que el conteo de esporas en el método grupal fue siempre mayor, en agosto la cantidad de esporas fue superior en el análisis individual en la mitad de las colmenas. Por ejemplo, en las colmenas 34 y 72, el conteo de esporas en el grupal fue de 7,300,000 y 6,600,000 respectivamente, mientras que en el examen individual correspondió a 7,672,000 $\pm 2,350,598$ y 7,249,000 $\pm 2,386,419$ esporas por abeja.

Durante setiembre en el 50.0\% de las colmenas $(1,28,39,40$ y 81) se observó un nivel de infección similar en ambos métodos. Ninguna de las colmenas mostró una intensidad fuerte. La colmena 1 tuvo la mayor cantidad de esporas, siendo el conteo individual superior al grupal, correspondiendo ambos procedimientos a semifuerte. Se debe tomar en cuenta que, durante el examen individual, algunas abejas presentaron más de 20,000,000 de esporas, lo que aumentó el promedio. Igualmente, en esta colmena se dio la mayor coincidencia del nivel de infección de abejas individuales con respecto a la muestra grupal, ya que en nueve de ellas fue el mismo (Figura 4). Por otra parte, en la mitad de las colmenas hubo diferencias en la intensidad de 
la infección. En la colmena 72, se contabilizó en el método grupal 6,100,000 esporas y el individual registró $991,666 \pm 620,447$, ubicándose en las categorías moderado y muy leve, respectivamente. En este caso, se presentó una diferencia considerable en el nivel de infección entre ambas técnicas, ya que corresponden a niveles no consecutivos.

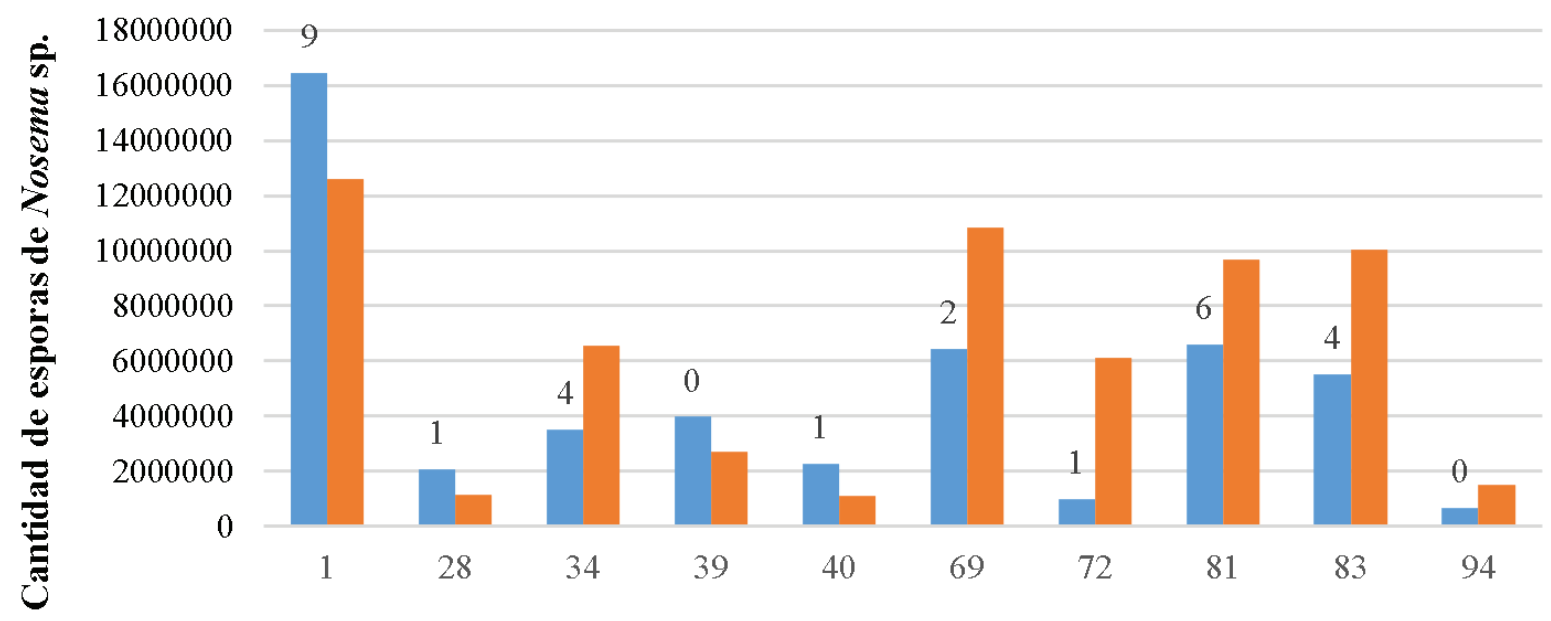

Colmenas muestreadas en setiembre

घ Análisis individual — Análisis grupal

Figura 4. Cantidad de esporas e intensidad de la infección de Nosema spp. en el examen grupal e individual de abejas obreras, durante el mes de setiembre. Se reporta el número de abejas individuales que mostró el mismo nivel de infección que la muestra grupal. Elaboración propia.

En octubre, el número de esporas disminuyó en la mayoría de las colmenas. Al comparar ambos métodos, se observó niveles de infección similares en tres colmenas (39, 40 y 83), cuyos valores obtenidos pertenecen a leve y moderado. Por otra parte, algunas colmenas presentaron diferencias en la intensidad de la infección según el método de análisis, sin embargo, no son considerables, ya que la cantidad de esporas se clasificó en niveles sucesivos. Por ejemplo, en la colmena 81 el conteo individual fue 9,132,000 \pm 1,988,020 (moderado), mientras que el grupal correspondió a 11,350,000 (semifuerte). Durante este mes, la mayor diferencia se observó en una colmena, en la que el análisis grupal resultó en 21,650,000 (fuerte) y con el procedimiento individual se encontró 4,769,666 $\pm 1,354,087$ de esporas (leve).

En noviembre, se cuantificó la menor cantidad de esporas, ninguna colmena mostró más de 10,000,000, resultando niveles de infección muy leve, leve y moderado. En la mayoría, el número de esporas fue superior en el análisis grupal. En la colmena 72, el resultado grupal fue de 3,600,000, mientras que el individual correspondió a 3,337,000 $\pm 1,090,014$, ambos con una intensidad leve. No obstante, en la colmena 40, el análisis individual fue 2,140,333 $\pm 930,563$ de esporas y en el examen grupal presentó 750,000, ubicándose como leve y muy leve, respectivamente. Asimismo, el mayor contraste fue en la colmena 39, con una diferencia de 5,938,334 de esporas por abeja, mientras que en la 72 se estableció la menor diferencia, siendo de 263,000 esporas. 


\section{Análisis de correlación}

Al comparar el número de esporas de Nosema spp. en los métodos individual y grupal por colmena de julio a noviembre, mediante análisis de correlación lineal, se observa que las cantidades obtenidas son similares para ambos procedimientos. La mayoría de los puntos trazados se encuentran cerca de la línea de tendencia, lo que indica la aproximación de los valores obtenidos por colmena en ambas técnicas de conteo de esporas (Figura 5). Además, el coeficiente de variación de 0.90, muestra que no hay diferencias significativas entre los métodos de análisis grupal e individual. Asimismo, se observa que el $\mathrm{R}^{2}$ de 0.80 establece un nivel confiable de eficiencia de ambos métodos en la determinación del número de esporas de nosemosis en abejas adultas.

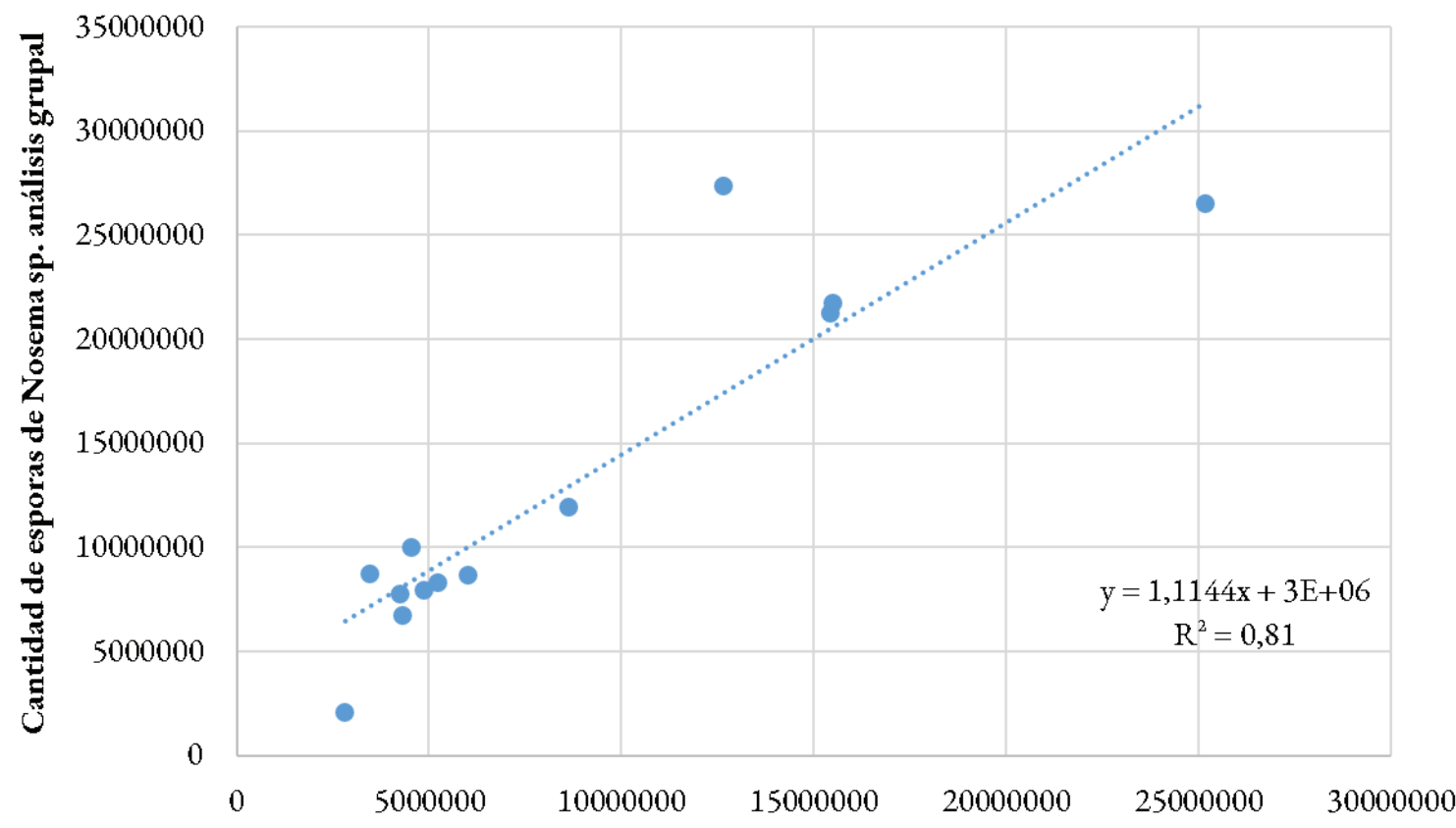

Cantidad de esporas de Nosema sp. análisis individual

Figura 5. Correlación de la cantidad de esporas de Nosema spp. determinadas en los métodos grupal e individual en abejas obreras adultas ( $\mathrm{R}^{2}$ de 0.80 , significancia de 0.05$)$. Cada punto corresponde a la cantidad promedio de esporas en ambos procedimientos para cada colmena durante el periodo de estudio. Elaboración propia.

\section{Discusión}

Como se indicó, la nosemosis es una de las enfermedades de mayor importancia económica en la apicultura mundial (Calderón \& Sánchez, 2007). Diversos estudios realizados en España indican un aumento en la pérdida de colmenas infectadas con N. ceranae (Botías et al., 2012). En la presente investigación, se evaluó el número de esporas y el nivel de infección en abejas infectadas naturalmente. Lo anterior es similar a una investigación desarrollada en España, donde las colmenas adquirieron el microsporidio de manera natural 
(Botías et al., 2013). En otros ensayos, las abejas se infectaron artificialmente con una cantidad específica de esporas (Huang et al., 2015).

\section{Nosema spp. en muestras grupales e individuales de abejas}

En este estudio realizado con abejas africanizadas, se determinó la presencia de nosemosis en todas las colmenas (prevalencia $=100.0 \%$ ), tanto en el análisis grupal como en el individual, con diferentes niveles de infección, desde muy leves hasta fuertes. Reportes similares en Italia (Papini et al., 2017) y en Canadá(Emsen et al., 2015) mencionan distintas intensidades de Nosema spp. en las colmenas examinadas.

Algunos factores que favorecen la nosemosis están relacionados con la ingesta de esporas al limpiar panales contaminados, así como a largos periodos de encierro de las abejas en la colmena (hacinamiento), ocasionados por vientos fuertes y lluvias persistentes. Entre más extensa la duración del hacinamiento, mayor es la cantidad de esporas, ya que los niveles de infección se elevan de manera considerable debido al contacto estrecho entre las abejas. Según Ritter (2001) se ha reportado que panales viejos en la cámara de cría, propician la presencia de esporas en la colmena. En el presente ensayo, se observaron panales muy oscuros en las colmenas. Panales en estas condiciones, con mucho tiempo de no cambiarse, pueden ser una fuente de contaminación de Nosema spp., ya que las esporas permanecen viables en ellos hasta por un año (DeGrandi-Hoffman \& Chen, 2015; Vivas, 2015).

El mayor conteo de esporas en ambos métodos durante la época lluviosa se registró en julio. Una de las posibles causas podría estar relacionada con el hacinamiento de las abejas debido a lluvias prolongadas. Aun cuando julio no fue el mes con mayor precipitación $(302 \mathrm{~mm})$, en algunas ocasiones se presentaron lluvias persistentes desde la mañana, las cuales permanecieron durante todo el día. Debido a que las abejas en estas condiciones no pueden defecar fuera de la colmena y al estrecho contacto entre ellas, probablemente favoreció la diseminación del microsporidio. Se menciona que las abejas jóvenes adquieren las esporas cuando realizan actividades de limpieza en panales contaminados (Martínez et al., 2011; Pacheco, 2008 ).

En los meses siguientes (agosto-octubre), hubo un descenso en la cantidad de esporas con respecto a julio, tanto en el análisis grupal como en el individual. Lo anterior representa un cambio considerable respecto a lo esperado, ya que las condiciones de precipitación y humedad, las cuales favorecen la nosemosis, aumentaron en los meses de setiembre (650 mm, 90.4\%) y octubre (540 mm, 90.6\%) (IMN, 2017). Las causas de esta disminución no se conocen con exactitud, no obstante, se debe mencionar que durante estos meses se presentaron las condiciones propias de la época, con mañanas soleadas y tardes lluviosas. Esta condición posiblemente permitió que las abejas realizaran sus actividades fuera de la colmena durante horas tempranas del día, como el pecoreo y sobre todo la defecación. Estos resultados coinciden con los reportados por Tapia et al. (2017) en Brasil, con abejas africanizadas, donde determinaron una disminución en el conteo de esporas durante la estación lluviosa comparado con la época seca. Igualmente, Emsen et al. (2015) indicaron bajos niveles de infección de Nosema spp. durante el invierno, los cuales aumentaron en el verano. Por otro lado, Retschnig et al. (2017) indican que la mayor intensidad del microsporidio se presentó en invierno.

En noviembre, se registró con ambos métodos el conteo más bajo de esporas. Se debe señalar que durante este periodo, se observó una reducción en la cantidad de lluvia, iniciando la transición hacia la época seca, con las condiciones más bajas de precipitación (126.2 mm) y humedad (87.4\%) (IMN, 2017). Lo anterior coincide con Calderón \& Ramírez (2010), quienes indican que, a menor cantidad de lluvia, el número de esporas de Nosema spp. disminuye en las colmenas. 


\section{Comparación de la cantidad promedio de esporas de Nosema spp.}

A la fecha se han realizado pocos estudios que comparen los niveles de nosemosis en muestras grupales e individuales de abejas de la misma colmena. Como se señaló, el método de laboratorio tradicional para el diagnóstico consiste en el examen de una muestra grupal de abejas adultas por colmena, mediante la que se obtiene un estimado del número de esporas por abeja (Botías et al., 2012; Prendas et al., 2018). Sin embargo, al aplicar este procedimiento podría obtenerse diferencias en el resultado, ya que la infección varía entre individuos de la misma colmena (Mulholland et al., 2012). El examen individual de abejas es más confiable para determinar la intensidad de la infección, sin embargo, es un procedimiento laborioso y requiere más tiempo que el método grupal. Pocas investigaciones mencionan la presencia de Nosema spp. en abejas individuales (Mulholland et al., 2012). Higes et al. (2008) indican que, en España, se han llevado a cabo algunos ensayos en abejas individuales de origen europeo.

En el presente ensayo, al comparar el número de esporas y el nivel de infección de Nosema spp. en una muestra grupal con respecto al análisis individual de abejas, provenientes de la misma colmena, se determinó una similitud en la tendencia del resultado, aun cuando el número de esporas, fue casi siempre mayor en el grupal. En ambos procedimientos se cuantificó la mayor cantidad de esporas en julio, mientras que en el mes de noviembre se registró la menor cantidad. Los resultados obtenidos permiten recomendar el análisis grupal de abejas, ya que es confiable para la detección y cuantificación del número de esporas del microsporidio Nosema spp.

\section{Intensidad mensual de Nosema spp. por colmena}

Durante julio, se observó la mayor diferencia en el número promedio de esporas (aproximadamente 8 millones), sin embargo, los niveles de infección fueron semejantes, ya que mediante el análisis grupal se clasificó como fuerte, mientras que el individual fue semifuerte. En este mes se observó el mayor número de abejas analizadas individualmente que presentaron el mismo nivel de infección que la muestra grupal. Lo anterior indica una notable similitud en el resultado obtenido en ambos métodos.

La estación lluviosa representa la época de escasez de alimento para las abejas, ya que las fuentes de néctar y polen son limitadas. Como lo mencionan Alux et al. (2010), existe una relación directa entre la nutrición y la inmunidad de las abejas, ya que, con una dieta abundante de polen, el sistema inmunológico responde adecuadamente contra la nosemosis. De acuerdo con Invernizzi et al. (2011) cuando aumenta la disponibilidad de polen, la infección por este microsporidio disminuye.

En los siguientes meses, agosto a octubre, hubo una reducción significativa en el número de esporas. Al comparar el análisis grupal e individual, se observó niveles de infección similares en más de la mitad de las colmenas, principalmente en los meses de agosto y setiembre. Por otra parte, en octubre se observó el mayor número de colmenas con diferencias en los niveles de infección según el método de análisis. No obstante, estas diferencias no fueron significativas, ya que el número de esporas se ubicó en niveles sucesivos. Según Mulholland et al. (2012), al analizar muestras grupales de abejas puede haber algunos individuos altamente infectados que alteran la cantidad promedio de esporas, lo que resulta en un incremento en la intensidad de infección de la muestra. Por lo anterior, conteos elevados de esporas no siempre corresponden a colonias enfermas, ya que en estos casos el análisis grupal de abejas puede sobrestimar la intensidad de la infección de la colonia.

En el mes de noviembre, se registró mediante ambos métodos el menor número de esporas, observándose 
muy leve, leve y moderado. Este descenso puede estar relacionado con la disminución de las lluvias y con temperaturas superiores a los $24^{\circ} \mathrm{C}$. Lo anterior, se relaciona con estudios realizados por Calderón \& Ramírez (2010); Calderón \& Sánchez (2011), donde mencionan que bajo condiciones de poca lluvia y temperaturas elevadas la prevalencia del microsporidio Nosema spp. tiende a disminuir. Chen et al. (2012) coinciden en indicar que a temperaturas cálidas este patógeno se encuentra en niveles bajos.

Se debe señalar que, para evitar resultados poco confiables, en la presente investigación se muestrearon únicamente abejas pecoreadoras (piquera). Estas abejas presentan mayores niveles de Nosema spp. que las nodrizas (ubicadas en el nido de cría), ya que el microsporidio ha tenido suficiente tiempo para multiplicarse, lo cual aumenta la posibilidad de observarlo en el laboratorio (Higes et al., 2008; Medina et al., 2014). Según Jack et al. (2016), muestras grupales colectadas de la cámara de cría, no deben considerarse para determinar la infección de una colmena, ya que presentan una combinación de abejas de distintas edades. Aun cuando las abejas adultas se colectaron homogéneamente de la piquera, al realizar el examen individual se observó variación tanto en el conteo de esporas, como en los niveles de infección. Lo cual puede estar relacionado con diferentes factores. Se ha indicado que en la colonia existen distintos tipos de barreras que permiten contrarrestar el efecto de un agente patógeno, las cuales son conocidas como inmunidad en conjunto. Esta inmunidad consiste en que cada individuo realiza labores que benefician a la colonia. Por ejemplo, las obreras mediante su comportamiento higiénico y de limpieza, remueven cría enferma y abejas muertas, eliminándolas de la colmena (Spivak, 1991). Además, algunos individuos que poseen niveles elevados de infección de Nosema spp. mueren cuando realizan actividades de pecoreo.

Adicionalmente, las abejas tienen varias líneas de defensa innata, como la barrera física, la que incluye el exoesqueleto y la membrana peritrófica (membrana de quitina y proteína) que recubre el sistema digestivo y actúa como una barrera, impidiendo el ingreso de microorganismos. Se ha reportado que cada abeja presenta características específicas que la hacen más o menos susceptible ante un agente infeccioso (Chupia et al., 2016). Una segunda línea de defensa basada en la inmunidad celular y humoral se activan si el patógeno sobrepasa las barreras físicas y químicas. Una nutrición adecuada sustentada en polen es necesaria para mantener un apropiado funcionamiento del sistema inmunológico de la abeja, que le permita responder de manera correcta ante una enfermedad como la nosemosis. Además, la variedad de microorganismos presentes en la composición microbiana del intestino de las abejas melíferas (microbiota), son de gran beneficio para contrarrestar las enfermedades (Biganski et al., 2017). Bacterias como Parasaccharibacter apium, disminuyen la intensidad de la infección de este microsporidio (DeGrandi-Hoffman \& Chen, 2015; Rubanov et al., 2019). Asimismo, en una investigación realizada en China, se determinó que la alimentación influye en la composición de la microbiota del intestino. Obreras alimentadas con pan de abeja (polen almacenado en las celdas) mostraron una mayor estabilidad en la microbiota y una menor tasa de mortalidad ante $N$. ceranae, comparado con abejas que se alimentaron con jarabe de azúcar (Huang et al., 2018).

\section{Comparación de los métodos de conteo de esporas mediante correlación}

Al realizar el análisis de correlación para comparar la cantidad de esporas de Nosema spp. obtenida por colmena de julio a noviembre, se observó que el resultado fue semejante para ambos métodos. La proximidad de los puntos a la línea de tendencia mostrada en el gráfico de correlación, indica la similitud del resultado entre ambos procedimientos para la cuantificación de esporas durante el periodo de estudio. Mulholland 
et al. (2012) indicaron un alto coeficiente de correlación entre la cantidad de abejas infectadas y el número promedio de esporas de $N$. apis por abeja en muestras grupales. También observaron una correlación similar entre los niveles de infección y el porcentaje de abejas infectadas, principalmente cuando los rangos de infección se ubicaron entre leve y moderado. En el presente estudio, los resultados más semejantes obtenidos en ambos métodos se observaron en noviembre, mes con los niveles más bajos de infección de nosemosis. Por otro lado, Jack et al. (2016) no encontraron una correlación entre la prevalencia y la intensidad del microsporidio durante la revisión de muestras grupales.

En conclusión, se determinó una alta prevalencia del microsporidio Nosema spp. durante la época lluviosa, con niveles desde muy leves hasta fuertes, tanto en el análisis grupal como en el individual. Al comparar la cantidad de esporas y la intensidad de la infección se obtuvo resultados similares en ambos procedimientos. Además, al correlacionar el número de esporas de Nosema spp. en muestras grupales e individuales de abejas por colmena durante los meses de estudio, se estableció proximidad de los valores, indicando cantidades similares. Lo anterior señala un resultado semejante tanto del examen grupal como del análisis individual de abejas con respecto al número de esporas y al nivel de infección de nosemosis de la misma colmena durante la época lluviosa.

\section{Agradecimientos}

Deseamos agradecer a nuestros compañeros M.Sc. Fernando Ramírez Arias y Tec. Guillermo Ramírez Arias (CINAT-UNA) por colaborar en el manejo general del apiario. Un agradecimiento al M.Sc. Walter Peraza Padilla (ECA-UNA) por la revisión preliminar del documento. Agradecemos a los estudiantes que apoyaron en la preparación de muestras de abejas adultas en el laboratorio.

\section{Referencias}

Alaux, C., Ducloz, F., Crauser, D. \& Le Conte, Y. (2010). Diet effects on honeybee immune competence. Biology Letters, 6, 562-565. https://doi.org/10.1098/rsbl.2009.0986.

Biganski, S., Kurze, C., Müller, M. \& Moritz, R. (2017). Social response of healthy honeybees towards Nosema ceranae - infected workers: care or kill? Apidologie, 49(3), 325-334. https://doi.org/10.1007/ s13592-017-0557-8.

Botías, C., Martín, R., Meana, A. \& Higes, M. (2012). Critical aspects of the Nosema spp. diagnostic sampling in honeybee (Apis mellifera L.) colonies. Parasitology Research, 110, 2557-2561. https://doi. org/10.1007/s00436-011-2760-2.

Botías, C., Martín, R., Barrios, L., Meana, A. \& Higes, M. (2013). Nosema spp. infection and its negative effects on honeybees (Apis mellifera iberiensis) at the colony level. Veterinary Research, 44, 25. https:// doi.org/10.1186/1297-9716-44-25.

Bravo, J., Carbonell, V., Valdebenedito, J. T., Figueroa, C., Valdovinos, C. E., Martín, R. \& Delporte, C. (2014). Identification of Nosema ceranae in the Valparaíso, Chile. Archivos de Medicina Veterinaria, 46(3), 487-491. http://dx.doi.org/10.4067/S0301-732X2014000300021. 
Calderón, R. A. \& Sánchez, L. A. (2007). Detección de enfermedades en abejas africanizadas en Costa Rica. Revista Ciencias Veterinarias, 25(2), 335-348. https://www.revistas.una.ac.cr/ index.php/veterinaria/ article/view/3676.

Calderón, R. A., Sánchez, L. A., Yañez, O. \& Fallas, N. (2008). Presence of Nosema ceranae in Africanized honey bee colonies in Costa Rica. Journal of Apicultural Research and Bee World, 47, 328-329. http:// dx.doi.org/10.1080/00218839.2008.11101485.

Calderón, R. A. \& Ramírez, F. (2010). Enfermedades de las abejas meliferas, con énfasis en abejas africanizadas. EUNA.

Calderón, R. A. \& Pichardo, J. (2011). Nosemiasis en abejas meliferas: diagnóstico, control y prevalencia. Publicaciones e Impresiones UNA.

Calderón, R. A. \& Sánchez, L. A. (2011). Diagnóstico de enfermedades en colmenas de abejas africanizadas en Costa Rica: prevalencia y distribución de setiembre a noviembre del 2007. Agronomía Costarricense, 35(2), 49-60. https://revistas.ucr.ac.cr/index.ph p/agrocost/article/view/6678.

Chemurot, M., De Smet, L., Brunain, M., De Rycke, R. \& de Graaf, D. (2017). Nosema neumanni (Microsporidia, Nosematidae), a new microsporidian parasite of honeybees, Apis mellifera in Uganda. European Journal of Protistology, 61, 13-19. https://doi.org/10.1016/j.ejop.2017.07.002.

Chen, Y., Chung, W., Wang, C., Solter, L. \& Huang, W. (2012). Nosema ceranae infection intensity highly correlates with temperature. Journal of Invertebrate Pathology, 111, 264-267. https://doi.org/10.1016/j. jip.2012.08.014.

Chihu, L., Chihu, D. \& Fernández, M. (2013). Nosema ceranae, un patógeno emergente en la apicultura mundial. Editorial Academia Española.

Chupia, V., Pikulkacw, S., Krutnuang, P., Mckchay, S. \& Patchancc, P. (2016). Molecular epidemology and geographical distribution of Nosema ceranae in honeybees, Northern Thailand. Asian Pacific Journal of Tropical Disease, 6(1), 27-31. https://doi.org/10.1016/S2222-1808(15)60980-X.

Copley, T., Chen, H., Giovenazzo, P., Houle, E. \& Jabaji, S. (2012). Prevalence and seasonality of Nosema species in Québec honeybees. The Canadian Entomologist, 144, 577-588. https://doi.org/10.4039/ tce.2012.46.

DeGrandi-Hoffman, G. \& Chen, Y. (2015). Nutrition, immunity and viral infections in honeybees. ScienceDirect, 10, 170-176. https://doi.org/10.1016/j.cois.2015.05.007.

Eiri, D., Suwannapong, G., Endler, M. \& Nieh, J. (2015). Nosema ceranae can infect honeybee larvae and reduces subsequent adult longevity. PLOS ONE, 10(5), 1-17. https://doi.org/10.1371/journal. pone.0126330.

Emsen, B., Guzman, E., Hamiduzzaman, M., Eccles, L., Lacey, B., Ruiz, R. \& Nasr, M. (2015). Higher prevalence and levels of Nosema ceranae than Nosema apis infections in Canadian honeybee colonies. Parasitology Research, 115(1), 175-181. https://doi.org/10.1007/s00436-015-4733-3. 
Foley, K., Fazio, G., Jensen, A. \& Hughes, W. (2014). The distribution of Aspergillus spp. opportunistic parasites in hives and their pathogenicity to honeybees. Veterinary Microbiology, 169(3-4), 203-210. https://doi.org/10.1016/j.vetmic.2013.11.029.

Fries, I. (2010). Nosema ceranae in European honeybees (Apis mellifera). Journal of Invertebrate Pathology, 103, S73-S79. https://doi.org/10.1016/j.jip.2009.06.017.

Hamiduzzaman, M., Guzmán, P. H., Goodwin, M., Reyes, G., Koleoglu, A., Correa, A. \& Petukhova, T. (2015). Differential responses of Africanized and European honeybees (Apis mellifera) to viral replication following mechanical transmission or Varroa destructor parasitism. Journal of Invertebrate Pathology, 126, 12-20. https://doi.org/10.1016/ j.jip.2014.12.004.

Higes, M., García, R., Martín, R. \& Meana, A. (2007). Experimental infection of Apis mellifera honeybees with Nosema ceranae (Microsporidia). Journal of Invertebrate Pathology, 94(3), 211-217. https://doi. org/10.1016/j.jip.2006.11.001.

Higes, M., Martín, R., Botías, C., Bailón, E. G., González, A. V., Barrios, L. \& Meana, A. (2008). How natural infection by Nosema ceranae causes honeybee colony collapse. Environmental Microbiology, 10(10), 2659-2669. https://doi.org/10.1111/j.1462-2920.2008.01687.x.

Higes, M., Martin, R. \& Meana, A. (2010). Nosema ceranae in Europe: an emergent type C Nosemosis. Apidologie, 41, 375-392. https://doi.org/10.1051/apido/2010019.

Huang, W., Solter, L., Aronstein, K. \& Huang, Z. (2015). Infectivity and virulence of Nosema ceranae and Nosema apis in commercially available North American honeybees. Journal of Invertebrate Pathology, 124, 107-113. https://doi.org/10.1016/j.jip.2014.10.006.

Huang, S., Ye, K., Huang, W., Ying, B., Su, X., Lin, L., Li, J., Chen, Y., Li, J., Bao, X. \& Hu, J. (2018). Influence of feeding type and Nosema ceranae infection on the gut microbiota of Apis cerana workers. American Society for Microbiology Systems, 3(6), 1-11. https://doi.org/10.1128/mSystems.00177-18.

Instituto Meteorológico Nacional. (2017). Regiones y subregiones climáticas del pais. https://www.imn.ac.cr/ publicaciones/estudios/Reg_climaCR.pdf.

Invernizzi, C., Santos, E., Garcia, E., Daners, G., Di Landro, R., Saadoun, A. \& Cabrera, C. (2011). Sanitary and nutritional characterization of honeybees colonies in Eucalyptus grandis plantations. Archivos de Zootecnia, 60, 1303-1314.

Jack, C., Lucas, H., Webster, T. \& Sagili, R. (2016). Colony level prevalence and intensity of Nosema ceranae in honeybees (Apis mellifera L.). PLOS ONE, 11(9), 1-20. https://doi.org/10.1371/journal. pone.0163522.

Jara, L., Muñoz, I., Cepero, A., Martín, R., Serrano, J., Higes, M. \& De la Rúa, P. (2015). Stable genetic diversity despite parasite and pathogen spread in honeybee colonies. The Science of Nature, 102, 53. https://doi.org/10.1007/s00114-015-1298-z.

Martínez, J., Medina, L. \& Catzín, A. (2011). Frecuencia de Varroa destructor, Nosema apis y Acarapis woodi en colonias manejadas y enjambres silvestres de abejas (Apis mellifera) en Mérida, Yucatán, México. Revista Mexicana de Ciencias Pecuarias, 2(1), 25-38. 
Medina, C., Guzmán, E., Espinosa, L., Uribe, L., Gutiérrez, R. \& Gutiérrez, F. (2014). Frecuencia de Varroosis y Nosemosis en colonias de abejas melíferas (Apis mellifera) en el estado de Zacatecas, México. Revista Chapingo Serie Ciencias Forestales y del Ambiente, 20(3), 30-36. https://doi.org/10.5154/r. rchscfa.2013.08.028.

Molina, A., Guzmán, E., Message, D., De Jong, D., Pesante, D., Mantilla, C. \& Meneses, G. (1990). Enfermedades y plagas de la abeja melifera occidental. OIRSA.

Mulholland, G., Traver, B., Johnson, N. \& Fell, R. (2012). Individual variability of Nosema ceranae infections in Apis mellifera colonies. Insects, 3, 1143-1155. https://doi.org/10.3390/insects3041143.

Muñoz, I., Cepero, A., Pinto, M., Martín, R., Higes, M. \& De la Rúa, P. (2014). Presence of Nosema ceranae associated with honeybee queen introductions. Infection, Genetics and Evolution, 23, 161-168. https:// doi.org/10.1016/j.meegid.2014.02.008.

Organización Mundial de Sanidad Animal (OIE). (2013). Manual de las pruebas de diagnóstico y las vacunas para los animales terrestres. http://www.oie.int/en/international-standard-setting/terrestrial-manual/ access-online/.

Organismo Internacional Regional de Sanidad Agropecuaria (OIRSA). (1990). Protocolo de técnicas laboratoriales de diagnóstico para enfermedades y plagas apicolas. http://portal.oirsa.org/contenido/ biblioteca/Protocolodetecnicaslaboratorialesdediagnosticodeenfermedadesyplagasapicolas.pdf.

Pacheco, L. (2008). Niveles de infección de Nosema apis Zander (Microspora: Nosematidae) en abejas adultas (Apis mellifera L.) y su relación con características del apicultor. Tesis de Pregrado,_Universidad Austral de Chile. Valdivia, Chile. http://cybertesis.uach.cl/tesis/uach/2008/fap116n/sources/fap116n.pdf.

Pacini, A., Giaocobino, A., Molineri, A., Bulacio, N., Aignasse, A., Zago, A. \& Signorini, M. (2016). Risk factors associated whith the abundance of Nosema spp. in apiaries located in temperate and subtropical conditions after honey harvest. Journal of Apicultural Research, 55(4), 342-350. https://doi.org/10.108 $\underline{0 / 00218839.2016 .1245396 .}$.

Papini, R., Manciati, F., Canovai, R., Cosci, F., Rocchigiani, G., Benelli, G. \& Canale, A. (2017). Prevalence of the microsporidian Nosema ceranae in honeybee (Apis mellifera) apiaries in Central Italy._Saudi Journal of Biological Sciences, 24(5), 979-982. http://dx.doi.org/10.1016/j.sjbs. 2017.01.010.

Pichardo, J., Calderón, R. A., van Veen, J. \& Ramírez, F. (2012). Nosemiasis en abejas Africanizadas: detección en cámara de cría y piquera. Notas Apicolas, 15, 26-33.

Prendas-Rojas, J., Figueroa-Mata, G., Ramírez-Montero, M., Calderón-Fallas, R. A., Ramírez-Bogantes, M. \& Travieso-González, C. (2018). Diagnóstico automático de infestación por Nosemiasis en abejas melíferas mediante procesado de imágenes. Revista Tecnología En Marcha, 31(2), 14-25. https://doi. org/10.18845/tm.v31i2.3621.

Retschnig, G., Williams, G., Schneeberger, A. \& Neumann, P. (2017)._Cold ambient temperature promotes Nosema spp. intensity in honey bees (Apis mellifera). Insects, 8, 20. https://doi.org/10.3390/ insects 8010020.

Ritter, W. (2001). Enfermedades de las abejas. Acribia S.A. 
Rubanov, A., Russell, K., Rothman, J., Nieh, J. \& McFrederick, Q. (2019). Intensity of Nosema ceranae infection is associated with specific honeybee gut bacteria and weakly associated with gut microbiome structure. Science Reports, 9, 1-8. https://doi.org/10.1038/s41598-019-40347-6.

Spivak, M. (1991). The Africanization process in Costa Rica. Westview Press Inc.

Tapia, J., Alcazar, G., Macías, J., Contreras, F., Tapia, J., Chavoya, F. \& Martínez, J. (2017). Nosemosis en abejas melíferas y su relación con factores ambientales en Jalisco, México. Revista Mexicana de Ciencias Pecuarias, 8, 3-4. http://dx.doi.org/10.22319/rmcp.v8i3.4510.

Traver, B. \& Fell, R. (2011). Prevalence and infection intensity of Nosema in honeybee (Apis mellifera L.) colonies in Virginia. Journal of Invertebrate Pathology, 107, 43-49. https://doi.org/10.1016/j. jip.2011.02.003.

Vivas, J. (2015). Prevalencia de Nosema (Nosema spp.) en colmenares de la región norte y centro norte del Ecuador. Tesis de grado, Universidad Central del Ecuador, Quito, Ecuador. 